\title{
Stratified random walks on the $n$-cube
}

\author{
F. R. K. Chung \\ University of Pennsylvania \\ Philadelphia, PA $19104^{1}$ \\ R. L. Graham \\ AT\&T Labs - Research \\ Murray Hill, NJ 07974
}

October 19,1996

\begin{abstract}
In this paper we present a method for analyzing a general class of ramdom walks on the $n$-cube (and certain subgraphs of it). These walks all have the property that the transition probabilities depend only on the level of the point the walk is at. For these walks, we derive sharp bounds on their mixing rates, i.e., the number of steps required to guarantee that the resulting distribution is close to the (uniform) stationary distribution.
\end{abstract}

\section{Introduction}

One popular object on which to study random walks is the so-called $n$-cube, denoted by $Q_{n}$ (see the next section for definitions). In this paper we present a method for analyzing a general class of random walks on the $n$-cube. These walks have the property that the transition probabilities (only) depend on the level (or weight) of the point the walk happens to be at, which is why we refer to them as stratified walks. All our walks will be reversible, and will have uniform stationary distributions. Our main goal will be to bound the rate at which the evolving distribution converges to its stationary distribution as a function of the number of steps taken by the walk. In particular, we will illustrate the method with several specific examples, giving for the first time sharp bounds on the mixing times of these walks.

For example, one special case is the following walk on $Q_{n} \backslash\{0\}$ (suggested by David Aldous [A94]): From $x=\left(x_{1}, \ldots, x_{n}\right) \in Q_{n} \backslash\{0\}$, choose a random pair $(i, j)$ of distinct indices and move to $x^{\prime}=\left(x_{1}^{\prime}, \ldots, x_{n}^{\prime}\right)$ where $x_{i}^{\prime} \equiv x_{i}+x_{j}(\bmod 2)$, and $x_{k}^{\prime}=x_{k}, k \neq i$. We show that $O(n \log n)$ steps suffice for this walk to approach its (uniform) stationary distribution.

\section{Preliminaries}

By the $n$-cube $Q_{n}$, we mean the graph with vertex set $V=\left\{\left(x_{1}, \ldots, x_{n}\right): x_{i}=0\right.$ or 1 , $1 \leq i \leq n\}$ and edge set $E$ consisting of all pairs of vertices $x, y \in V$ which differ in exactly 
one coordinate. We indicate edges by writing $x y \in E$, or $x \sim y$. The weight $w(x)$ of a vertex $x$ is just the number of coordinates which are equal to 1.

We will let $p=\left(p_{0}, p_{1}, \ldots, p_{n-1}\right)$ with $0<p_{i} \leq 1,0 \leq i \leq n-1$, denote the transition probability vector which will determine our process. It defines a walk $W$, which we call a $p$-walk on $Q_{n}$, as follows:

If the walk is currently at $x=\left(x_{1}, \ldots, x_{n}\right)$ with $w(x)=k$ then for the next step, select a random coordinate $i$ (each with probability $1 / n$ ), and then move to

$$
x^{\prime}=\left(x_{1}, \ldots, x_{i-1}, 1-x_{i}, x_{i+1}, \ldots, x_{n}\right) \text { with probability }\left\{\begin{array}{ll}
p_{k} & \text { if } x_{i}=0 \\
p_{k-1} & \text { if } x_{i}=1
\end{array},\right.
$$

and otherwise, do nothing. It is easy to see that this $p$-walk $W$ is reversible, and if not all the $p_{k}=1$ (which we will henceforth assume), the walk is aperiodic with a uniform stationary distribution. The standard random walk on $Q_{n}$ in which you either move to a random neighbor or stay put, all with equal probability $1 /(n+1)$, corresponds to the choice $p=\left(\frac{n}{n+1}, \frac{n}{n+1}, \ldots, \frac{n}{n+1}\right)$. For $x, y \in V$, let us write $x \subset y$ if $x_{i}=1 \Rightarrow y_{i}=1$.

It will be useful to write down the transition matrix $Q$ corresponding to the $p$-walk $W$. Thus, $Q$ is a $2^{n} \times 2^{n}$ matrix indexed by the $x \in V$ with $Q(x, y)$ denoting the probability of going from $y$ to $x$ in one step, and given by

$$
Q(x, y)= \begin{cases}\frac{1}{n} p_{k-1} & \text { if } w(x)=k-1, w(y)=k, x \subset y \\ \frac{1}{n} p_{k} & \text { if } w(x)=k+1, w(y)=k, y \subset x \\ 1-\frac{k}{n} p_{k-1}-\frac{(n-k)}{n} p_{k} & \text { if } x=y, w(x)=k \\ 0 & \text { otherwise } .\end{cases}
$$

\section{An overview}

Our plan for analyzing the $p$-walk $W$ will consist of the following steps:

(i) We decompose $\mathbb{E}^{2^{n}}$ into various invariant subspaces under the action of $Q$. This will result in the formation of smaller matrices $P_{0}, P_{1}, \ldots$, whose eigenvalues are just the eigenvalues of $Q$ (with appropriate multiplicities). Furthermore, the eigenvectors of $Q$ are all formed from the eigenvectors of the $P_{i}$ by simple linear transformations.

(ii) The largest eigenvalue of $Q$ is $\rho_{0}=1$, which will also be an eigenvalue of $P_{0}$. We then derive good estimates for the second largest eigenvalue $\rho_{1}$ of $P_{0}$, by relating $P_{0}$ to a random walk on a certain weighted path $G_{n}$. 
(iii) We next upper bound all the other eigenvalues of the $P_{i}, i \geq 1$ (most of which are substantially smaller than $\left.\rho_{1}\right)$.

(iv) We use hitting time arguments to show that the "central" points of $G_{n}$ are always hit fairly soon with high probability.

(v) We then bound the mixing time (using either total variation or relative pointwise distance) for approaching the stationary distribution on $G_{n}$, assuming that we have started from a central point.

(vi) We finally lift the results back up to our original walk on $Q_{n}$ to obtain the desired mixing time estimates for $W$.

Of course, to get precise answers, we must make some specific assumptions about $p$. We will do this for several particular examples, namely, when $p_{k}$ grows linearly in $k$, and when $p_{k}$ grows like $k^{\alpha}, \alpha>1$. Interpolation results can then be applied to treat the more general cases $p_{k}=O(k)$ and $p_{k}=O\left(k^{\alpha}\right), \alpha>1$. We remark that our techniques also apply to the simpler case that $p_{k}$ is constant. However, such walks have already been well-studied in the literature (e.g., see [LT79], [DGM90]) so we will not discuss them here.

\section{Decomposing $Q$}

Let us denote by $V_{k}$ the set of $\left(\begin{array}{l}n \\ k\end{array}\right)$ vectors $x \in V$ of weight $k, 0 \leq k \leq n$. We will identify a $k$-element subset $(=k$-set $)$ of $[n]:=\{1,2, \ldots, n\}$ with a weight $k$ vector $x$ in the usual way, namely

$$
x \longleftrightarrow\left\{i \in[n]: x_{i}=1\right\}
$$

(so that we can also think of $V_{k}$ as the set of $k$-sets of $[n]$ ). It will be convenient to regard $Q$ as being formed from blocks, induced by the $V_{k}$ in the natural way:

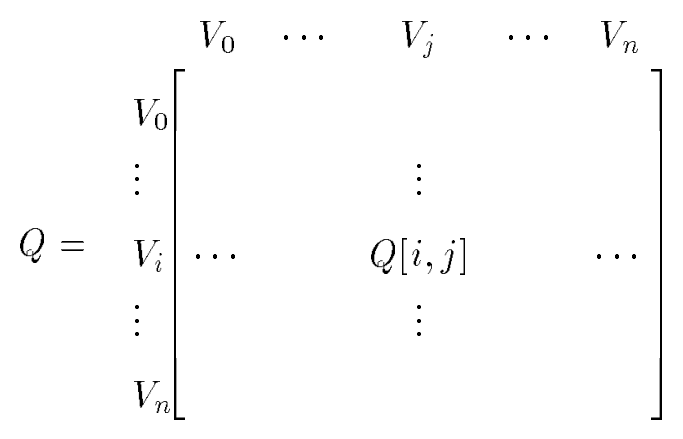

Here, $Q[i, j]$ is an $\left(\begin{array}{l}n \\ i\end{array}\right)$ by $\left(\begin{array}{l}n \\ j\end{array}\right)$ submatrix whose structure we now describe. 
Define $M_{i, j}, 0 \leq i, j \leq n$, to be the comparability matrix of the sets $V_{i}$ and $V_{j}$. That is, $M_{i, j}$ is indexed by $V_{i}$ and $V_{j}$, and for $x \in V_{i}, y \in V_{j}$,

$$
M_{i, j}(x, y)=\left\{\begin{array}{ll}
1 & \text { if } x \subset y \text { or } y \subset x \\
0 & \text { otherwise }
\end{array}\right. \text {. }
$$

Then it is easily seen that

$$
Q[i, j]=\left\{\begin{array}{ll}
\frac{p_{k}}{n} M_{k, k+1} & \text { if } i=k, j=k+1 \\
\frac{p_{k}}{n} M_{k+1, k} & \text { if } i=k+1, j=k \\
\left(1-\frac{k}{n} p_{k-1}-\frac{(n-k)}{n} p_{k}\right) M_{k, k} & \text { if } i=j=k \\
0 & \text { otherwise }
\end{array} .\right.
$$

We abbreviate:

$$
\begin{aligned}
q(k, k+1) & =q(k+1, k)=p_{k} / n \\
q(k, k) & =1-\frac{k}{n} p_{k-1}-\frac{(n-k)}{n} p_{k} .
\end{aligned}
$$

Since $M_{k, k}$ is just an $\left(\begin{array}{l}n \\ k\end{array}\right)$ by $\left(\begin{array}{l}n \\ k\end{array}\right)$ identity matrix, and $M_{k+1, k}=M_{k, k+1}^{*}$ (with * denoting transpose) then we see that $Q$ is symmetric.

Our next goal will be to separate the eigenvectors and eigenvalues of $Q$.

To begin, suppose $X=\left(X_{0}, X_{1}, \ldots, X_{n}\right)^{*}$ satisfies the eigenvalue equation

$$
Q X=\rho X
$$

where $X_{k}$ is a block of $X$ of length $\left(\begin{array}{l}n \\ k\end{array}\right)$, and has the form

$$
X_{k}=c_{k} M_{k, n i} y_{k}
$$

for some real numbers $y_{k}$ and constants $c_{k}$ (which will be determined shortly). Define

$$
M_{n}:=\left(\begin{array}{ccccc}
c_{0} M_{n, 0} & & & & \\
& c_{1} M_{n, 1} & & 0 & \\
& & \ddots & & \\
& 0 & & \ddots & \\
& & & & c_{n} M_{n, n}
\end{array}\right)
$$

and $Y=\left(y_{0}, y_{1}, \ldots, y_{n}\right)^{*}$. Thus, $X=M_{n}^{*} Y$. It follows by (1) that

$$
M_{n} Q M_{n}^{*} Y=\rho M_{n} M_{n}^{*} Y
$$


Let us now choose the $c_{k}$ so that $M_{n} M_{n}^{*}=I$ (an appropriate identity matrix). Hence,

$$
c_{k}^{2} M_{n k} M_{k n}=I
$$

which implies

$$
c_{k}=\left(\begin{array}{l}
n \\
k
\end{array}\right)^{-1 / 2}, 0 \leq k \leq n .
$$

Therefore, setting

$$
P_{0}:=M_{n} Q M_{n}^{*}
$$

we have by (3)

$$
P_{0} Y=\rho Y
$$

so that the $n+1$ eigenvalues of $P_{0}$ are also eigenvalues of $Q$. In fact, if $Y$ is an eigenvector of $P_{0}$ with eigenvalue $\rho$, then $X=M_{n}^{*} Y$ is an eigenvector of $Q$ with eigenvalue $\rho$. Direct computation shows that:

$$
\begin{aligned}
P_{0}(k, k) & =1-p_{k-1} \cdot \frac{k}{n}-p_{k} \frac{(n-k)}{n}=q(k, k) \\
P_{0}(k, k+1) & =P_{0}(k+1, k)=\sqrt{(k+1)(n-k)} \frac{p_{k}}{n}, 0 \leq k \leq n,
\end{aligned}
$$

and all other entries are 0 .

Now suppose that $X^{\prime}$ is an eigenvector of $Q$ which does not arise from those of $P_{0}$ in this way. Then clearly $X^{\prime} \in \operatorname{ker} M_{n}$. For the next step, assume $X^{\prime} \in \operatorname{ker} M_{n}$ satisfies (1), and has the form

$$
X^{\prime}=\left(0, X_{1}^{\prime}, X_{2}^{\prime}, \ldots, X_{n-1}^{\prime}, 0\right)^{*}
$$

where $X_{k}^{\prime}$ is a block of $X^{\prime}$ of length $\left(\begin{array}{l}n \\ k\end{array}\right)$ and has the form

$$
X_{k}^{\prime}=c_{k}^{\prime} M_{k, n-1} y_{k}^{\prime}, 1 \leq k \leq n-1,
$$

for some $y_{k}^{\prime}$ (vectors indexed by $(n-1)$ )-sets of $[n]$ ) and constants $c_{k}^{\prime}$ (which will be determined shortly). Define

$$
M_{n-1}:=\left(\begin{array}{ccccc}
0 & & & & \\
& c_{1}^{\prime} M_{n-1,1} & & & 0 \\
& & \ddots & & \\
& & & \ddots & \\
& & & & c_{n-1}^{\prime} M_{n-1, n-1} \\
& & & & 0
\end{array}\right)
$$


and

$$
Y^{\prime}=\left(0, y_{1}^{\prime}, \ldots, y_{n-1}^{\prime}, 0\right)^{*}
$$

As before, (1) implies

$$
M_{n-1} Q M_{n-1}^{*} Y^{\prime}=\rho M_{n-1} M_{n-1}^{*} Y^{\prime} .
$$

Now it is easily checked that

$$
M_{n-1, k} M_{k, n-1}=\left(\begin{array}{c}
n-2 \\
k-1
\end{array}\right) M_{n-1, n-1}+\left(\begin{array}{c}
n-2 \\
k
\end{array}\right) M_{n-1, n} M_{n, n-1}, 1 \leq k \leq n-1 .
$$

Hence,

$$
\begin{aligned}
\left(c_{k}^{\prime}\right)^{2} M_{n-1, k} M_{k, n-1} y_{k}^{\prime} & =\left(c_{k}^{\prime}\right)^{2}\left(\begin{array}{l}
n-2 \\
k-1
\end{array}\right) y_{k}^{\prime}+c_{k}^{\prime}\left(\begin{array}{c}
n-2 \\
k
\end{array}\right) M_{n-1, n}\left(c_{k}^{\prime} M_{n, n-1} y_{k}^{\prime}\right) \\
& =\left(c_{k}^{\prime}\right)^{2}\left(\begin{array}{l}
n-2 \\
k-1
\end{array}\right) y_{k}^{\prime}
\end{aligned}
$$

since

$$
\begin{aligned}
c_{k}^{\prime}\left(\begin{array}{c}
n-1 \\
k
\end{array}\right) M_{n, n-1} y_{k}^{\prime} & =c_{k}^{\prime} M_{n, k} M_{k, n-1} y_{k}^{\prime} \\
& =M_{n, k} X_{k}^{\prime}=0
\end{aligned}
$$

by the assumption that $X^{\prime} \in \operatorname{ker} M_{n}$. We now choose

$$
c_{k}^{\prime}=\left(\begin{array}{l}
n-2 \\
k-1
\end{array}\right)^{-1 / 2}
$$

which then gives by (7)

$$
M_{n-1} Q M_{n-1}^{*} Y^{\prime}=\rho Y^{\prime}
$$

Consider the matrix

$$
\bar{P}_{1}:=M_{n-1} Q M_{n-1}^{*}
$$

which we can regard as a block matrix by deleting the first and last rows and columns (which are all zero). Then

$$
\bar{P}_{1}(k, k)=c_{k}^{\prime} M_{n-1, k} q(k, k) c_{k}^{\prime} M_{k, n-1}=q(k, k) I .
$$

where $I$ is an $\left(\begin{array}{c}n \\ n-1\end{array}\right) \times\left(\begin{array}{c}n \\ n-1\end{array}\right)$ identity matrix. Since by (8),

$$
c_{k}^{\prime} M_{n-1, k} q(k, k+1) M_{k, k+1} c_{k+1}^{\prime} M_{k+1, n-1}=\frac{p_{k}}{n} \sqrt{k(n-k-1)} I+T
$$


where $T v=0$ for $v \in \operatorname{ker} M_{n, n-1}$. Now define the $(n-1) \times(n-1)$ matrix $P_{1}$ by choosing

$$
\begin{aligned}
P_{1}(k, k) & =q(k, k) \\
P_{1}(k, k+1)=P_{1}(k+1, k) & =\frac{p_{k}}{n} \sqrt{k(n-k-1)},
\end{aligned}
$$

for $1 \leq k \leq n-1$.

In addition, if $Z^{\prime}=\left(z_{1}, z_{2}, \ldots, z_{n-1}\right)^{*}$ satisfies

$$
P_{1} Z^{\prime}=\rho Z^{\prime}
$$

and $v \in \operatorname{ker} M_{n, n-1}$ then

$$
X_{k}^{\prime}=M_{k, n-1} v z_{k}
$$

defines an eigenvector $X^{\prime}=\left(0, X_{1}^{\prime}, \ldots, X_{n-1}^{\prime}, 0\right)^{*}$ of $Q$ in ker $M_{n}$ with eigenvalue $\rho$. Since

$$
\operatorname{dim}\left(\operatorname{ker} M_{n, n-1}\right)=\left(\begin{array}{l}
n \\
1
\end{array}\right)-\left(\begin{array}{l}
n \\
0
\end{array}\right)=n-1
$$

then for each eigenvalue $\rho$ in (10), we can produce $n-1$ independent eigenvectors $X^{\prime}$ for $Q$ with eigenvalue $\rho$.

We will carry out the preceding argument repeatedly to describe all the eigenvectors and eigenvalues of $Q$. In general, we assume that for a fixed $j, X \in \operatorname{ker} M_{n-j+1}$ satisfies (1), and has the form

$$
X=\left(0, \ldots, 0, X_{j}, \ldots, X_{n-j}, 0, \ldots, 0\right)^{*}
$$

where $X_{k}$ is a block of $X$ of length $\left(\begin{array}{l}n \\ k\end{array}\right)$ and has the form

$$
X_{k}=c_{k} M_{k, n-j} y_{k}, j \leq k \leq n-j,
$$

for some $y_{k}$ (vectors indexed by $\left(n-j\right.$ )-sets of $[n]$ ) and constants $c_{k}$ (which will be determined shortly).

Note that $\operatorname{ker} M_{n-j+1} \subset \operatorname{ker} M_{l}$ for $l \geq n-j+1$. 
Define

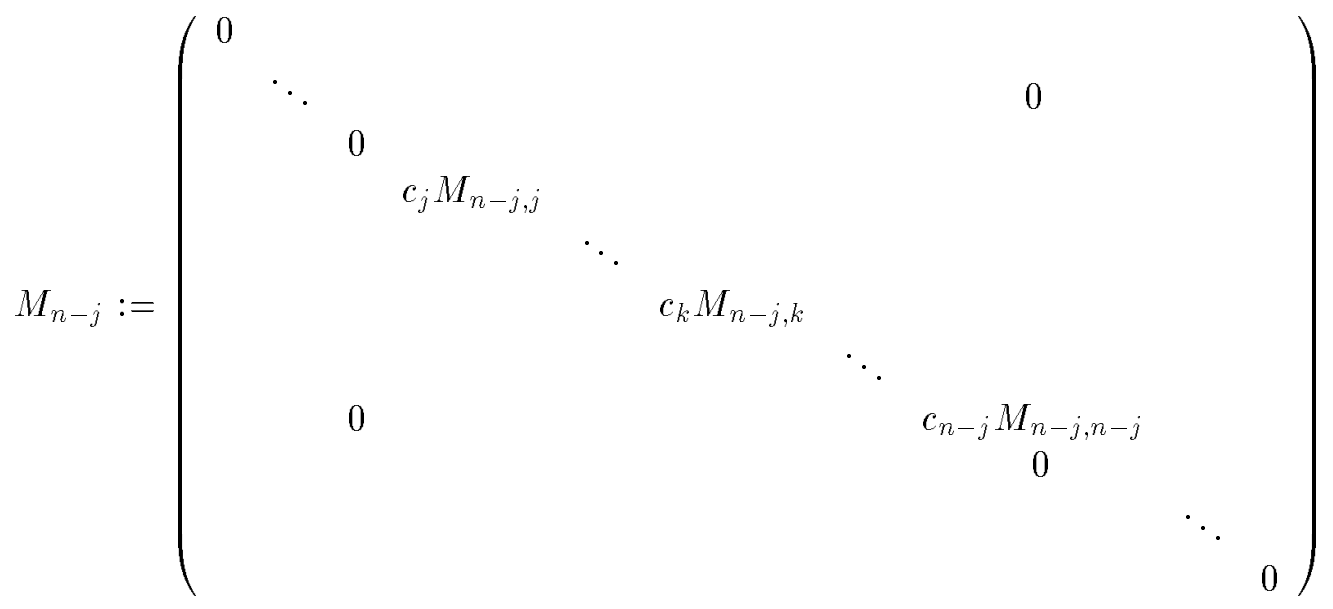

and

$$
Y=\left(0, \ldots, 0, y_{j}, \ldots, y_{n-j}, 0, \ldots, 0\right)^{*}
$$

By (1) we have

$$
M_{n-j} Q M_{n-j}^{*} Y=\rho M_{n-j} M_{n-j}^{*} Y .
$$

It can be checked that

$$
M_{n-j, k} M_{k, n-j}=\left(\begin{array}{c}
n-2 j \\
n-j-k
\end{array}\right) M_{n-j, n-j}+\sum_{\ell>n-j} c_{\ell}^{\prime \prime} M_{n-j, \ell} M_{\ell, n-j}
$$

for $n-j \leq k \leq j$ and for appropriate constants $c_{\ell}^{\prime \prime}$. Therefore, we have

$$
\begin{aligned}
\left(c_{k}\right)^{2} M_{n-j, k} M_{k, n-j} y_{k} & =\left(c_{k}\right)^{2}\left(\begin{array}{c}
n-2 j \\
n-j-k
\end{array}\right) y_{k}+\sum_{\ell>n-j} c_{\ell}^{\prime \prime} M_{n-j, \ell}\left(c_{k} M_{\ell, n-j} y_{k}\right) \\
& =\left(c_{k}\right)^{2}\left(\begin{array}{c}
n-2 j \\
n-j-k
\end{array}\right) y_{k}
\end{aligned}
$$

since

$$
\begin{aligned}
c_{\ell}^{\prime \prime}\left(\begin{array}{c}
n-j \\
k
\end{array}\right) M_{\ell, n-j} y_{k} & =c_{\ell}^{\prime \prime} M_{\ell, k} M_{k, n-j} y_{k} \\
& =M_{\ell, k} X_{k}=0
\end{aligned}
$$

for $X \in \operatorname{ker} M_{n-j+1} \subset \operatorname{ker} M_{\ell}$. We now specify

$$
c_{k}=\left(\begin{array}{c}
n-2 j \\
n-j-k
\end{array}\right)^{-1 / 2}
$$

Then

$$
M_{n-j} Q M_{n-j}^{*} Y=\rho Y .
$$


Let

$$
\bar{P}_{j}:=M_{n-j} Q M_{n-j}^{*}
$$

which we can regard as a block matrix by deleting the first and last $j$ rows and columns (which are all zero). The $(k, k)$-block of $\bar{P}_{j}$, for $j \leq k \leq n-j$, is given by

$$
\bar{P}_{j}(k, k)=c_{k} M_{n-j, k} q(k, k) M_{k, k} c_{k} M_{k, n-j}=q(k, k) I
$$

where $I$ is an $\left(\begin{array}{c}n \\ n-j\end{array}\right) \times\left(\begin{array}{c}n \\ n-j\end{array}\right)$ identity matrix.

To compute the $(k, k+1)$ and $(k+1, k)$ blocks of $\bar{P}_{j}$, using (11) we have

$$
c_{k} M_{n-j, k} q(k, k+1) M_{k, k+1} c_{k+1} M_{k+1, n-j}=\frac{p_{k}}{n} \sqrt{(n-j-k)(k-j+1)} I+T
$$

where $T v=0$ for $v \in \operatorname{ker} M_{n-j+1, n-j}$. Now, define the $(n-2 j+1) \times(n-2 j+1)$ matrix $P_{j}$ by choosing

$$
\begin{aligned}
P_{j}(k, k) & =q(k, k) \\
P_{j}(k, k+1)=P_{j}(k+1, k) & =\sqrt{(k-j+1)(n-j-k)} \frac{p_{k}}{n} .
\end{aligned}
$$

for $j \leq k \leq n-j$.

Hence, if $Z=\left(z_{j}, \ldots, z_{n-j}\right)^{*}$ satisfies

$$
P_{j} Z=\rho Z
$$

and $v \in \operatorname{ker} M_{n-j+1, n-j}$ then

$$
X_{k}=M_{k, n-j} v z_{k}
$$

defines an eigenvector $X=\left(0, \ldots, 0, X_{j}, \ldots, X_{n-j}, 0, \ldots, 0\right)$ of $Q$ in $\operatorname{ker} M_{n-j+1}$ with eigenvalue $\rho$. Since

$$
\operatorname{dim}\left(\operatorname{ker} M_{n-j+1, n-j}\right)=\left(\begin{array}{c}
n \\
n-j
\end{array}\right)-\left(\begin{array}{c}
n \\
n-j+1
\end{array}\right)
$$

then for each eigenvalue $\rho$ in (12) we can produce $\left(\begin{array}{c}n \\ n-j\end{array}\right)-\left(\begin{array}{c}n \\ n-j+1\end{array}\right)$ independent eigenvectors $X$ for $Q$ with eigenvalue $\rho$.

Executing this process for $1 \leq j \leq\left\lfloor\frac{n}{2}\right\rfloor$, and noting that $P_{j}$ has $n-2 j+1$ independent eigenvectors, then the total number of eigenvectors of $Q$ generated this way (counting the $n+1$ from $\left.P_{0}\right)$ is just

$$
n+1+\sum_{j=1}^{\left\lfloor\frac{n}{2}\right\rfloor}(n-2 j+1)\left(\left(\begin{array}{c}
n \\
n-j
\end{array}\right)-\left(\begin{array}{c}
n \\
n-j+1
\end{array}\right)\right)=2^{n}
$$

and so we have found a complete set. 


\section{The Aldous cube}

We will now examine in some detail a particular example of a $\rho$-walk $W_{A}$ which will illustrate more specifically the approach we have described. This walk $W_{A}$, in which $x_{i}$ is replaced by $x_{i}+x_{j}(\bmod 2)$ for a randomly chosen pair $(i, j)$ of distinct indices (see the description at the end of Section 1) corresponds to the choice $p_{k}=\frac{k}{n-1}, 0 \leq k \leq n-1$. Note that this is actually a walk on the punctured cube $Q_{n}^{-}=Q_{n} \backslash\{0\}$. Thus, in this case we have

$$
\begin{aligned}
q(k, k) & =1-k / n \text { and } \\
P_{j}(k, k+1)=P_{j}(k+1, k) & =\frac{k}{n(n-1)} \sqrt{(k-j+1)(n-k-j)},
\end{aligned}
$$

for $j \leq k \leq n-j$, and $0 \leq j \leq\left\lfloor\frac{n}{2}\right\rfloor$.

As usual, the largest eigenvalue of $P_{0}$ is 1 . We will show that any other eigenvalue $\rho^{\prime}$ of $P_{0}$ satisfies

$$
\rho^{\prime} \leq 1-\frac{1}{3 n}
$$

Furthermore, all other eigenvalues of $Q_{A}$ (the name for the transition matrix $Q$ in this case) are significantly smaller than this. To prove (14), we first define

$$
P_{0}^{\prime}:=I-P_{0}
$$

where $I$ is an $n \times n$ identity matrix. Thus,

$$
\begin{aligned}
P_{0}^{\prime}(k, k) & =1-P_{0}(k, k), \\
P_{0}^{\prime}(k, k+1) & =P_{0}^{\prime}(k+1, k)=-P_{0}(k, k+1)
\end{aligned}
$$

and all other entries are zero.

Claim. $P_{0}^{\prime}$ is the (normalized) Laplacian matrix of a weighted path $G_{n}$ on a set $[n]=$ $\{1,2, \ldots, n\}$ of $n$ vertices. The degree $d_{k}$ of vertex $k$ is $n(n-1)\left(\begin{array}{l}n \\ k\end{array}\right)$. The edge weight $w_{k}$ on the edge $\{k, k+1\}$ is $k(n-k)\left(\begin{array}{l}n \\ k\end{array}\right)$. (Thus, the loop weight at $k$ is $(n-1)(n-k)\left(\begin{array}{l}n \\ k\end{array}\right)$.) This claim follows by direct verification from the definition of the Laplacian for $G_{n}$ (see [C96], [CY95] for background material on Laplacians on graphs).

In fact, $G_{n}$ is just obtained from $Q_{n} \backslash\{0\}$ by collapsing all vertices of weight $k$ in $Q_{n} \backslash\{0\}$ into the single vertex $k$ of $G_{n}$, for $1 \leq k \leq n$. 
Thus, for any $g:[n] \rightarrow \mathbb{R}$,

$$
\begin{aligned}
P_{0}^{\prime} g(x) & =\frac{1}{\sqrt{d_{x}}} \sum_{\substack{y \\
y \sim x}}\left(\frac{g(x)}{\sqrt{d_{x}}}-\frac{g(y)}{\sqrt{d_{y}}}\right) w_{x y} \\
& =\frac{1}{\sqrt{d_{x}}} \sum_{\substack{y \\
y \sim x}}(f(x)-f(y)) w_{x y}
\end{aligned}
$$

where

$$
\begin{aligned}
& f(x):=g(x) / \sqrt{d_{x}}, x \in[n] \\
& d_{x}=n(n-1)\left(\begin{array}{l}
n \\
x
\end{array}\right), x \in[n]
\end{aligned}
$$

and

$$
w_{x y} \text { is the edge weight on the edge } x y \text {. }
$$

(Of course, for the path $G_{n}, w_{x y}=0$ unless $|x-y|=1$.) If we denote the eigenvalues of $P_{0}^{\prime}$ by

$$
0=\lambda_{0}<\lambda_{1} \leq \lambda_{2} \leq \cdots \leq \lambda_{n}
$$

then our first goal is to lower-bound $\lambda_{1}$. We will do this by constructing a "nearby" weighted path $\hat{G}_{n}$ for which we can control $\hat{\lambda}_{1}=\hat{\lambda}_{1}\left(\hat{P}_{0}\right)$ and its corresponding eigenfunction exactly, and then applying a comparison theorem for relating $\lambda_{1}$ to $\hat{\lambda}_{1}$.

The weighted path $\hat{G}_{n}$ will have the same vertex set $[n]$ as $G_{n}$. The degrees in $\hat{G}_{n}$ are given by

$$
\hat{d}_{k}=\left\{\begin{array}{ll}
n^{2}\left(n^{2}-1\right) /(n-3) & \text { for } \quad k=1 \\
n(n+1)\left(\begin{array}{l}
n \\
k
\end{array}\right) & \text { for } \quad 2 \leq k \leq n
\end{array} .\right.
$$

(We are assuming $n>3$.) The edge weights $\hat{w}_{k}$ for the edges $\{k, k+1\}$ of $\hat{G}_{n}$ are given by

$$
\hat{w}_{k}=(k+2)(n-k)\left(\begin{array}{l}
n \\
k
\end{array}\right), 1 \leq k \leq n-1 .
$$

Define $\hat{f}:[n] \rightarrow \mathbb{R}$ by

$$
\hat{f}(k):=\frac{1}{k+1}-\frac{2}{n+1}, 1 \leq k \leq n
$$

and set

$$
\hat{g}(k):=\hat{f}(k) \sqrt{d_{k}} .
$$


We claim that $\hat{\lambda}_{1}=\frac{1}{n}$ is the smallest positive eigenvalue of the corresponding Laplacian matrix $\hat{P}_{0}$ for $\hat{G}_{n}$, and that $\hat{g}$ is its corresponding eigenvector. To see this, we first must check (which is straightforward) that $\hat{f}$ satisfies the following condition (analogous to (15)):

$$
\hat{P}_{0} \hat{g}(x)=\frac{1}{\sqrt{d_{x}}} \sum_{\substack{y \\ y \sim x}}(\hat{f}(x)-\hat{f}(y)) \hat{w}_{x y}=\frac{1}{n} \hat{g}(x)
$$

for all $x \in[n]$. This can be rewritten as

$$
\frac{1}{n} \hat{d}_{k} \hat{f}(k)=(\hat{f}(k)-\hat{f}(k+1)) \hat{w}_{k}+(\hat{f}(k)-\hat{f}(k-1)) \hat{w}_{k-1}
$$

for all $k \in[n]$, where we take $\hat{w}_{0}=\hat{w}_{n}=0$.

We note that the eigenvalue $\hat{\lambda}_{0}=0$ of $\hat{P}_{0}$ has the eigenvector $\hat{g}_{0}(k)=\sqrt{d_{k}}, k \in[n]$. It is easily checked that $\hat{g}$ is orthogonal to $\hat{g}_{0}$. We also note from (18) that $\hat{f}$ is monotone (which will soon be needed).

Next, we argue that any eigenvalue $\lambda$ of $\hat{P}_{0}$ with an eigenfunction $g$ for which $f(k)=$ $g(k) / \sqrt{d_{k}}$ is not monotone must satisfy $\lambda>\hat{\lambda}_{1}$. To do this, we use the following characterization of $\hat{\lambda}_{1}$ (cf. [C96]):

$$
\hat{\lambda}_{1}=\inf _{h \neq 0} \sup _{c} \frac{\sum_{k=1}^{n-1}(h(k)-h(k+1))^{2} \hat{w}_{k}}{\sum_{k=1}^{n}(h(k)-c)^{2} \hat{d}_{k}} .
$$

So, let us assume that $\lambda$ is an eigenvalue of $\hat{P}_{0}$ with an associated eigenvector $g$ for which $f(k)=g(k) / \sqrt{\hat{d}_{k}}$ is not monotone.

Claim. $\lambda>\hat{\lambda}_{1}$.

Proof. Define

$$
f^{\prime}(k)=\sum_{j=2}^{k}|f(j)-f(j-1)|, k \geq 2 .
$$

Choose $c_{0}$ so that

$$
\sum_{k=1}^{n}\left(f^{\prime}(k)-c_{0}\right) \hat{d}_{k}=0 .
$$

Also, choose $i_{0}$ so that $f^{\prime}\left(i_{0}\right) \leq c_{0}<f^{\prime}\left(i_{0}+1\right.$ ). (If no such $i_{0}$ exists then $f^{\prime}$ and $f$ must be constant, which is a contradiction.) Without loss of generality, we can assume that $f\left(i_{0}\right) \leq$ $f\left(i_{0}+1\right)$. Now, define

$$
c^{\prime}=f^{\prime}\left(i_{0}\right)-f\left(i_{0}\right) .
$$




\section{Fact.}

$$
\left|f^{\prime}(k)-c_{0}\right| \geq\left|f(k)-c_{0}+c^{\prime}\right| \text { for } \quad 1 \leq k \leq n
$$

Proof of (24). For $k=i_{0},(24)$ holds with equality by (23). Also

$$
\begin{aligned}
f^{\prime}\left(i_{0}+1\right)-c_{0} & =f^{\prime}\left(i_{0}\right)+\left|f\left(i_{0}+1\right)-f\left(i_{0}\right)\right|-c_{0} \\
& =f\left(i_{0}\right)-c_{0}+c^{\prime}+f\left(i_{0}+1\right)-f\left(i_{0}\right) \\
& =f\left(i_{0}+1\right)-c_{0}+c^{\prime}
\end{aligned}
$$

which implies (24) for $k=i_{0}+1$.

Now, in general for $k>i_{0}+1$,

$$
\begin{aligned}
\left|f^{\prime}(k)-c_{0}\right| & =|| f(k)-f(k-1)\left|+f^{\prime}(k-1)-c_{0}\right| \\
& =|| f(k)-f(k-1)|+\cdots+| f\left(i_{0}+2\right)-f\left(i_{0}+1\right)\left|+f^{\prime}\left(i_{0}+1\right)-c_{0}\right| \\
& \geq|| f(k)-f\left(i_{0}+1\right)\left|+f\left(i_{0}+1\right)-c_{0}+c^{\prime}\right| \\
& \geq\left|f(k)-c_{0}+c^{\prime}\right|
\end{aligned}
$$

as required by (24). Similarly, for $k<i_{0}$,

$$
\begin{aligned}
\left|f^{\prime}(k)-c_{0}\right| & =\left|f^{\prime}(k+1)-\right| f(k)-f(k+1)\left|-c_{0}\right| \\
& =\left|f^{\prime}\left(i_{0}\right)-c_{0}-\right| f\left(i_{0}-1\right)-f\left(i_{0}\right)|-\cdots-| f(k)-f(k+1)|| \\
& \geq\left|f^{\prime}\left(i_{0}\right)-\right| f(k)-f\left(i_{0}\right)\left|-c_{0}\right| \\
& \geq\left|f\left(i_{0}\right)-c_{0}+c^{\prime}+f(k)-f\left(i_{0}\right)\right| \\
& =\left|f(k)-c_{0}+c^{\prime}\right| .
\end{aligned}
$$

This proves (24).

Note that since by assumption $f$ is not monotone, then strict equality must hold in (24) for some $k$. Setting $c_{1}=c_{0}-c^{\prime}$, we obtain

$$
\sum_{k}\left(f^{\prime}(k)-c_{0}\right)^{2} \hat{d}_{k} \geq \sum_{k}\left(f(k)-c_{1}\right)^{2} \hat{d}_{k}
$$

It follows from the preceding argument that equality holds in (25) if and only if

$$
f^{\prime}(k)=f(k)-c_{1}+c_{0} \text { for all } k
$$


Now, by (20) we have

$$
\begin{aligned}
\lambda & =\sup _{c} \frac{\sum_{k=1}^{n-1}(f(k)-f(k+1))^{2} \hat{w}_{k}}{\sum_{k=1}^{n}(f(k)-c)^{2} \hat{d}_{k}} \\
& \geq \frac{\sum_{k=1}^{n-1}(f(k)-f(k+1))^{2} \hat{w}_{k}}{\sum_{k=1}^{n}\left(f(k)-c_{1}\right)^{2} \hat{d}_{k}} \\
> & \frac{\sum_{k=1}^{n-1}\left(f^{\prime}(k)-f^{\prime}(k+1)\right)^{2} \hat{w}_{k}}{\sum_{k=1}^{n}\left(f^{\prime}(k)-c_{0}\right)^{2} \hat{d}_{k}}
\end{aligned}
$$

by (25) since $f$ is not monotone

$$
\begin{aligned}
& \geq \inf _{h \neq 0} \sup _{c^{\prime}} \frac{\sum_{k=1}^{n-1}(h(k)-h(k+1))^{2} \hat{w}_{k}}{\sum_{k=1}^{n}\left(h(k)-c^{\prime}\right)^{2} \hat{d}_{k}} \\
& =\hat{\lambda}_{1} .
\end{aligned}
$$

This proves the Claim.

Next, we claim that $\hat{P}_{0}$ cannot have two different eigenvectors $g_{1}$ and $g_{2}$, both orthogonal to $g_{0}$ and to each other, so that the corresponding functions $f_{1}(k)=g_{1}(k) / \sqrt{\hat{d}_{k}}$ and $f_{2}(k)=$ $g_{2}(k) / \sqrt{\hat{d}_{k}}$ are both monotone.

To see this, will expand our $n$-vectors back to $N$-tuples by the mapping

$$
f \rightarrow F=(\overbrace{f(1), \ldots, f(1)}^{\left(\begin{array}{l}
n \\
1
\end{array}\right)}, \ldots, \overbrace{f(k), \ldots, f(k)}^{\left(\begin{array}{c}
n \\
k
\end{array}\right)}, \ldots, \overbrace{f(n)}^{\left(\begin{array}{l}
n \\
n
\end{array}\right)=1}),
$$

(where $N=2^{n}-1$ ). So, assume to the contrary that $F_{1}$ and $F_{2}$ are both monotone (w.l.o.g. increasing) and

$$
\left\langle F_{1}, 1\right\rangle=\sum_{k=1}^{N} F_{1}(k)=0=\left\langle F_{2}, 1\right\rangle=\left\langle F_{1}, F_{2}\right\rangle
$$

where $F_{1} \not \equiv 0, F_{2} \not \equiv 0$, and 1 is the all 1 's vector.

It well known (see [GKP94]) that the permutation $\pi$ on $[N]=\{1,2, \ldots, N\}$ which maximizes $\sum_{i=1}^{N} F_{1}(i) F_{2}(\pi(i))$ is the choice $\pi=$ identity on $[N]$. Hence, by $(26)$ we have

$$
\sum_{i=1}^{N} F_{1}(i) F_{2}(\sigma(i)) \leq 0 \text { for every permutation } \sigma \text { on }[N] \text {. }
$$


Therefore,

$$
\begin{aligned}
0 & =\sum_{i=1}^{N} F_{1}(i) \sum_{j=1}^{N} F_{2}(j)=\sum_{i, j=1}^{N} F_{1}(i) F_{2}(j) \\
& =\sum_{i=1}^{N} \sum_{j=1}^{N} F_{1}(i+j) F_{2}(i+\sigma(j))
\end{aligned}
$$

(where addition inside $F_{k}$ is taken modulo $N$ ) which implies

$$
\sum_{i=1}^{N} F_{1}(i) F_{2}(\sigma(i))=0 \text { for every permutation } \sigma \text { on }[N] \text {. }
$$

In particular, this implies

$$
\begin{aligned}
& F_{1}(1) F_{2}(1)+F_{1}(N) F_{2}(N)+\sum_{i=2}^{N-1} F_{1}(i) F_{2}(i) \\
= & F_{1}(1) F_{2}(N)+F_{1}(N) F_{2}(1)+\sum_{i=2}^{N-1} F_{1}(i) F_{2}(i)
\end{aligned}
$$

i.e.,

$$
F_{1}(1) F_{2}(1)+F_{1}(N) F_{2}(N)=F_{1}(1) F_{2}(N)+F_{1}(N) F_{2}(1)
$$

or

$$
\left(F_{1}(N)-F_{1}(1)\right)\left(F_{2}(N)-F_{2}(1)\right)=0 .
$$

However, this implies either $F_{1}$ or $F_{2}$ is constant, which is impossible, and our claim is proved.

As a result of the preceding remarks, we can finally conclude that the smallest nonzero eigenvalue $\hat{\lambda}_{1}$ of $\hat{P}_{0}$ satisfies

$$
\hat{\lambda}_{1}=1 / n
$$

Our next job will be to establish the following:

Comparison Lemma. Suppose $P$ and $P^{\prime}$ are two weighted paths on $[n]$ with degrees $d_{i}$ and $d_{i}^{\prime}$, and edge weights $w_{i}$ and $w_{i}^{\prime}$, respectively. Assume that for all $i$ we have

$$
d_{i} \geq \alpha d_{i}^{\prime}, w_{i}^{\prime} \geq \beta w_{i}
$$

Then

$$
\lambda_{1}^{\prime} \geq \alpha \beta \lambda_{1}
$$


where $\lambda_{1}$ and $\lambda_{1}^{\prime}$ are the smallest positive eigenvalues of the associated Laplacians on $P$ and $P^{\prime}$, respectively.

We give a short proof for completeness. (The reader can consult [C96] for more general versions.) Let $f^{\prime}$ denote a so-called "harmonic" eigenvector of $P^{\prime}$ associated with $\lambda_{1}^{\prime}$ (i.e., $g^{\prime}(k)=f^{\prime}(k) \sqrt{d_{k}^{\prime}}$ is an eigenvector of $P^{\prime}$ with eigenvalue $\left.\lambda_{1}^{\prime}\right)$. Considering the Rayleigh quotient (see $(20)$ ), we have

$$
\begin{aligned}
\lambda_{1}^{\prime} & \geq \frac{\sum_{i}\left(f^{\prime}(i)-f^{\prime}(i+1)\right)^{2} w_{i}^{\prime}}{\sum_{i}\left(f^{\prime}(i)-c_{0}\right)^{2} d_{i}^{\prime}} \\
& \geq \frac{\alpha \beta \sum_{i}\left(f^{\prime}(i)-f^{\prime}(i+1)\right)^{2} w_{i}}{\sum_{i}\left(f^{\prime}(i)-c_{0}\right)^{2} d_{i}} \\
& \geq \alpha \beta \lambda_{1}
\end{aligned}
$$

where $c_{0}$ is chosen so that $\sum_{i}\left(f^{\prime}(i)-c_{0}\right) d_{i}=0$ (thus minimizing the denominator). This proves $(31)$.

Finally, we apply (31) with $\hat{P}_{0}$ and $P_{0}^{\prime}$ taking the roles of $P$ and $P^{\prime}$, respectively. From (16) and (17) we find

$$
\frac{\hat{d}_{i}}{d_{i}} \geq \frac{n+1}{n-1}, \frac{w_{i}}{\hat{w}_{i}} \geq \frac{w_{1}}{\hat{w}_{1}}=\frac{1}{3} \text { for all } i
$$

Therefore,

$$
\lambda_{1} \geq \frac{1}{3}\left(\frac{n+1}{n-1}\right) \hat{\lambda}_{1}=\frac{n+1}{3 n(n-1)}>\frac{1}{3 n} .
$$

This implies

$$
\rho_{1} \leq 1-\frac{n+1}{3 n(n-1)}
$$

which is slightly stronger than (14).

Our next goal will be to bound all the other eigenvalues of the other $P_{k}, k \geq 1$. We will do this by using the fact that any eigenvalue of a nonnegative matrix is bounded above in absolute value by the maximum row sum of the matrix. It follows from the general expressions for $P_{k}(i, j)$ at the end of Section 4 that the $i^{\text {th }}$ row sum $\sigma(k, i)$ of $P_{k}$ is

$\sigma(k, i)=\frac{1}{n(n-1)}\{(n-1)(n-i)+i \sqrt{(i-k+1)(n-k-i)}+(i-1) \sqrt{(i-k)(n-k-i+1)}\}$

for $k \leq i \leq n-k$. 


\section{Claim.}

$$
\sigma(k, i) \leq 1-\frac{k}{2 n}
$$

Proof. Note that

$$
\begin{aligned}
i \sqrt{(i-k+1)(n-k-i)} & =\sqrt{i(i-k+1) i(n-k-i)} \\
& \leq \sqrt{\left(\frac{2 i-k+1}{2}\right)^{2}\left(\frac{n-k}{2}\right)^{2}} \\
& =\frac{1}{4}(2 i-k+1)(n-k)
\end{aligned}
$$

Therefore,

$$
\begin{aligned}
\sigma(k, i) & \leq \frac{1}{n(n-1)}\left\{(n-1)(n-i)+\frac{1}{4}(2 i-k+1)(n-k)+\frac{1}{4}(2 i-k-1)(n-k)\right\} \\
& =\frac{1}{n(n-1)}\left\{(n-1)(n-i)+(n-k)\left(i-\frac{k}{2}\right)\right\} \\
& \leq \frac{1}{n}\left(n-\frac{k}{2}\right)
\end{aligned}
$$

which proves (34).

As a consequence, any eigenvalue $\rho^{(k)}$ of $P_{k}, k \geq 1$, satisfies

$$
\left|\rho^{(k)}\right| \leq 1-\frac{k}{2 n}
$$

Our next job will be to bound the expected time for the walk on $G_{n}$ to hit the vertex $\left\lfloor\frac{n}{2}\right\rfloor$, given that we start at vertex 1 . In general, let $E_{i} T_{i+1}$ denote the expected number of steps it takes to reach vertex $i+1$, given that we start at vertex $i$. Then it is not hard to show (e.g., see Aldous [A93]):

$$
E_{i} T_{i+1}=\frac{1}{w_{i}} \sum_{j=1}^{i} d_{j}
$$

which in turn implies

$$
E_{1} T_{n / 2}=E_{1} T_{2}+E_{2} T_{3}+\cdots+E_{n / 2-1} T_{n / 2}
$$

where $n / 2$ will denote $\lfloor n / 2\rfloor$ when $n$ is odd.

\section{Claim.}

$$
E_{1} T_{n / 2}<3 n \log n \quad \text { for } \quad n \geq 2
$$




\section{Proof.}

$$
\begin{aligned}
E_{1} T_{n / 2} & =\sum_{i=1}^{n / 2-1} \sum_{j=1}^{i} \frac{d_{j}}{w_{i}} \\
& =\sum_{i=1}^{\frac{n}{2}-1} \sum_{j=1}^{i} \frac{n(n-1)\left(\begin{array}{c}
n \\
j
\end{array}\right)}{i(n-i)\left(\begin{array}{l}
n \\
i
\end{array}\right)} \\
& \leq n(n-1) \sum_{i=1}^{\frac{n}{2}-1} \frac{1}{i(n-i)} \sum_{j=1}^{i}\left(\frac{i}{n-i}\right)^{i-j} \\
\leq & \operatorname{since} \frac{\left(\begin{array}{l}
n \\
j
\end{array}\right)}{\left(\begin{array}{c}
n \\
i
\end{array}\right)} \leq\left(\frac{i}{n-i}\right)^{i-j} \text { for } j \leq i \\
& =n(n-1) \sum_{i=1}^{\frac{n}{2}-1} \frac{1}{i(n-i)} \frac{1}{1-\frac{i}{n-i}} \\
& =2(n-1) \sum_{i=1}^{\frac{n}{2}-1}\left(\frac{1}{i(n-2 i)}+\frac{1}{n-2 i}\right) \\
& <3 n \log n \cdot \mathbf{m}
\end{aligned}
$$

A similar argument shows that the expected time to hit the vertex $n / 2$ starting from the other end of $G_{n}$, namely, from the vertex $n$, can be bounded by the same quantity (in fact, a somewhat smaller quantity. We omit the proof.) It therefore follows that for any $i \in[n]$,

$$
E_{i} T_{n / 2}<3 n \log n
$$

A key step now will be to bound the mixing time of our $p$-walk assuming that we are allowed to start from some vertex $y_{0} \in V_{n / 2}$ (i.e., the binary $n$-tuple $y_{0}$ has weight $n / 2$ ). By symmetry, all vertices in $V_{n / 2}$ have the same behavior. Thus, we need to bound

$$
\begin{aligned}
2 \Delta_{T V}\left(s, y_{0}\right) & :=\sum_{x \in V}\left|Q_{A}^{s}\left(y_{0}, x\right)-\pi(x)\right| \\
\leq \Delta^{\prime}\left(s, y_{0}\right) & :=\left(\sum_{x \in V} \frac{\left(Q_{A}^{s}\left(y_{0}, x\right)-1 / N\right)^{2}}{1 / N}\right)^{1 / 2} .
\end{aligned}
$$

where $\Delta_{T V}$ denotes the total variation distance (but starting at $y_{0}$ ), $N=2^{n}-1$ and $\pi(x)=1 / N$ is the uniform (stationary) distribution on $V$.

Let $\delta_{y_{0}}: V \rightarrow \mathbb{R}$ denote the characteristic function of $y_{0}$, i.e.,

$$
\delta_{y_{0}}(x)=\left\{\begin{array}{ll}
1 & \text { if } x=y_{0} \\
0 & \text { otherwise }
\end{array} .\right.
$$


Then we can write

$$
\delta_{y_{0}}=\sum_{i} \phi_{i}\left(y_{0}\right) \phi_{i}
$$

where the $\phi_{i}$ denote orthonormal eigenfunctions for $Q_{A}$, and $\phi_{0}$ corresponds to the eigenvalue 0 . Let $I_{0}$ denote the operator which projects a function defined on $V$ to the eigenspace generated by $\phi_{0}$, i.e., if $f=\sum_{i} a_{i} \phi_{i}$ then $I_{0} f=a_{0} \phi_{0}$. Then

$$
\begin{aligned}
\sum_{x}\left(Q_{A}^{s}\left(y_{0}, x\right)-1 / N\right)^{2} & =\sum_{x}\left(\delta_{y_{0}}\left(Q_{A}^{s}-I_{0}\right) \delta_{x}^{*}\right)^{2} \\
& =\sum_{x} \delta_{y_{0}}\left(Q_{A}^{s}-I_{0}\right) \delta_{x}^{*} \delta_{x}\left(Q_{A}^{s}-I_{0}\right) \delta_{y_{0}}^{*} \\
& =\delta_{y_{0}}\left(Q_{A}^{2 s}-I_{0}\right) \delta_{y_{0}}^{*} \\
& =\sum_{i \neq 0} \rho_{i}^{2 s} \phi_{i}^{2}\left(y_{0}\right)
\end{aligned}
$$

where $1=\rho_{0}>\rho_{1} \geq \ldots \geq \rho_{N-1}$ are the eigenvalues of $Q_{A}$. Note that

$$
\sum_{y \in V} \phi_{i}^{2}(y)=1 \text { for all } i
$$

Therefore

$$
\sum_{y \in V_{n / 2}}\left(\Delta^{\prime}(s, y)\right)^{2} \leq \sum_{i \neq 0} \sum_{y \in V} \phi_{i}^{2}(y) \rho_{i}^{2 s}=N \sum_{i \neq 0} \rho_{i}^{2 s}
$$

Since

$$
\sum_{y \in V_{n / 2}}\left(\Delta^{\prime}(s, y)\right)^{2}=\left(\begin{array}{c}
n \\
n / 2
\end{array}\right)\left(\Delta^{\prime}\left(s, y_{0}\right)\right)^{2}
$$

then we obtain

$$
\left(\Delta^{\prime}\left(s, y_{0}\right)\right)^{2} \leq \frac{N}{\left(\begin{array}{c}
n \\
n / 2
\end{array}\right)} \sum_{i \neq 0} \rho_{i}^{2 s} \leq 2 \sqrt{n} \sum_{i \neq 0} \rho_{i}^{2 s} .
$$

Finally, we need to bound the right-hand side of (45).

Claim. If $s \geq 4 n \log n+c n$ then

$$
\Delta^{\prime}\left(s, y_{0}\right) \leq \sqrt{\frac{2}{n}}\left(\frac{1}{e^{c}-1}+\frac{1}{e^{2 c / 3}}\right)^{1 / 2} .
$$


Proof. By (35) , (45), (32) and the remarks following (12), we have

$$
\begin{aligned}
\left(\Delta^{\prime}\left(s, y_{0}\right)\right)^{2} & \leq 2 \sqrt{n}\left\{n\left(1-\frac{1}{3 n}\right)^{2 s}+\sum_{k=1}^{\lfloor n / 2\rfloor}(n-2 k+1)\left(\left(\begin{array}{l}
n \\
k
\end{array}\right)-\left(\begin{array}{c}
n \\
k-1
\end{array}\right)\right)\left(1-\frac{k}{2 n}\right)^{2 s}\right\} \\
& \leq 2 \sqrt{n}\left\{\exp \left(\log n-\frac{2 s}{3 n}\right)+\sum_{k=1}^{\lfloor n / 2\rfloor} \exp \left(\log (n-2 k+1)+k \log n-\frac{s k}{n}\right)\right\} \\
& \leq 2 \sqrt{n}\left(n^{-5 / 3} e^{-2 c / 3}+\frac{1}{n^{2}} \sum_{k=1}^{\lfloor n / 2\rfloor} e^{-c k}\right) \\
& \leq \frac{2}{n}\left(e^{-2 c / 3}+\left(e^{c}-1\right)^{-1}\right)
\end{aligned}
$$

which proves (46).

We now have all the ingredients necessary for our final estimates.

If $S_{i}$ denotes the numbers of steps taken starting at vertex $i$ in $G_{n}$ until vertex $n / 2$ is first reached then by $(39)$

$$
E\left[S_{i}\right]:=\mu_{i}<3 n \log n .
$$

Hence,

$$
\operatorname{Pr}\left[S_{i} \geq 2 \mu_{i}\right] \leq 1 / 2
$$

and, more generally, for any positive integer $t$,

$$
\operatorname{Pr}\left[S_{i} \geq 2 t \mu\right] \leq \operatorname{Pr}\left[S_{i} \geq 6 \operatorname{tn} \log n\right] \leq 2^{-t} .
$$

Thus, for the total variation distance $\Delta_{T V}$ defined by

$$
\Delta_{T V}(s):=\sup _{y \in V} \Delta_{T V}(s, y)
$$

then by (46) and (47) we have

$$
\begin{aligned}
\Delta_{T V}(s) \leq & \frac{1}{2^{t}}+\frac{1}{\sqrt{n}}\left(\frac{1}{n^{c}-1}+\frac{1}{n^{2 c / 3}}\right)^{1 / 2} \\
& \text { if } \quad s \geq(6 t+4+c) n \log n .
\end{aligned}
$$

This implies the simpler (but weaker) result:

$$
\Delta_{T V}(s) \leq 2^{2-a / 9} \quad \text { if } \quad s \geq a n \log n
$$

It may in fact be true that $\Delta_{T V}(s) \rightarrow 0$ for $s \geq c_{0} n \log n$ with a fixed constant $c_{0}$, as $n \rightarrow \infty$. This would follow if we knew that for some fixed $c_{1}, \operatorname{Pr}\left[S_{1} \geq c_{1} n \log n\right] \rightarrow 0$ as $n \rightarrow \infty($ see $(47))$. 
We point out that for the standard $p$-walk on $Q_{n}$ having $p_{k}=n /(n+1)$ for all $k$, the mixing time is known (see [DGM90]) to be of the form $\frac{1}{4} n \log n+c n$. Hence, it is impressive (to us) that the walk $W_{A}$ also has a mixing time of order $O(n \log n)$, given that in this case it is much harder to leave points of low weight.

We also note that earlier preliminary results ([DS96], [CG96]) established a bound of order $O\left(n^{2} \log n\right)$ on the mixing time on $W_{A}$.

We close this section with some remarks on another common metric on probability distributions. This is the relative pointwise distance $\Delta(s)$ of $P^{s}$ to its stationary distribution $\pi$, given by

$$
\Delta(s):=\max _{x, y \in V} \frac{\left|P^{s}(y, x)-\pi(x)\right|}{\pi(x)} .
$$

It turns out that for the walk $W_{A}$ on the Aldous cube, at least $s=c n^{2}$ steps are required to force $\Delta(s) \rightarrow 0$. To see this, let $x_{0} \in V_{1}$ be a vertex of weight 1 . Of course,

$$
\Delta(s) \geq \frac{\left|Q_{A}^{s}\left(x_{0}, x_{0}\right)-\pi\left(x_{0}\right)\right|}{\pi\left(x_{0}\right)} .
$$

Since $p_{1}=1 /(n-1)$ for $W_{A}$, then for any distribution $f$,

$$
f Q_{A}\left(x_{0}\right) \geq\left(\frac{n-2}{n-1}\right) f(x),
$$

and this implies

$$
\delta_{x} Q_{A}^{s}(x) \geq\left(\frac{n-2}{n-1}\right)^{s}
$$

Thus,

$$
\Delta(s) \geq\left|\left(2^{n}-1\right)\left(\frac{n-2}{n-1}\right)^{s}-1\right| .
$$

This implies in particular that for $s \leq n^{2} \log 2-n, \Delta(s)$ is bounded away from 0 .

On the other hand, the following argument shows that $n^{2}$ is the correct order of growth. This will follow from the following fact (which applies to the standard random walk $P$ on any regular weighted graph $G$ ).

Fact. The mixing time under relative pointwise distance can be at most a factor of $O(\log N)$ times the mixing time under total variation distance (where $N=|G|$ ).

Proof. Standard arguments (e.g., see [C96]) show that

$$
\begin{aligned}
\Delta_{T V}(s) & \leq \Delta(s) \leq e^{-s \lambda_{1}} \frac{\operatorname{vol} G}{\min _{x} d_{x}}=N e^{-s \lambda_{1}} \leq \epsilon \\
& \text { if } \quad s \geq \frac{1}{\lambda_{1}} \log \frac{N}{\epsilon}
\end{aligned}
$$


where vol $G:=\sum_{x} d_{x}$

On the other hand,

$$
\begin{aligned}
\Delta_{T V}(s) & =\max _{y} \max _{A \subseteq V}\left|\sum_{x \in A} P^{s}(y, x)-\pi(x)\right| \\
& \geq \sup _{f} \max _{A \subseteq V}\left|\sum_{x \in A}\left(f P^{s}(x)-\pi(x)\right)\right|
\end{aligned}
$$

over all initial probability distributions $f$. Let us choose $f T^{-1 / 2}=c \phi_{1}$, where $T$ is the diagonal matrix of degrees $d_{x}, \phi_{1}$ is an eigenfunction corresponding to the eigenvalue $\lambda_{1}$, and $c^{-1}=\sum_{x}\left|\phi_{1} T^{1 / 2}(x)\right|$. Then

$$
\begin{aligned}
\Delta_{T V}(s) & \geq c^{-1} \sum_{x}\left|f P^{s}(x)-\pi(x)\right| \\
& \geq c^{-1} \sum_{x}\left|\left(1-\lambda_{1}\right)^{s} \phi_{1} T^{1 / 2}(x)\right| \\
& =c^{-1}\left(1-\lambda_{1}\right)^{s} \sum_{x}\left|\phi_{1} T^{1 / 2}(x)\right| \\
& =\left(1-\lambda_{1}\right)^{s} .
\end{aligned}
$$

This shows that $\Delta_{T V}(s)$ is bounded away from 0 for any $s=c^{\prime} / \lambda_{1}, c^{\prime}$ a fixed constant. This, together with (49), completes the proof.

Applying (50) to the Aldous cube walk, where $\lambda_{1} \geq 1 / 3 n$, we get

$$
\Delta(s) \leq e^{-c / 3} \text { if } \quad s \geq 3 n^{2} \log 2+c n
$$

which shows that $c^{\prime} n^{2}$ is the correct order of growth for the mixing time under relative pointwise distance. It would be interesting to know what the correct coefficient of $n^{2}$ is, and whether this walk exhibits a cut-off phenomenon (cf. [DGM90]).

\section{Slower walks}

We now describe what happens when our $p$-walk has $p_{k}$ growing like $k^{\alpha}$ for some $\alpha>1$. Specifically, we will assume that

$$
p_{k}=\left(\frac{k+1}{n+1}\right)^{\alpha}, 0 \leq k \leq n
$$

where $\alpha>1$ is arbitrary but fixed. Note that in contrast to the Aldous cube situation, $p_{0}>0$, so all $2^{n}$ points participate in the walk. Since the argument follows the preceding procedure rather closely, we will only hint at the proofs, pointing out differences along the way. The bottom line is given by the following result. 
Theorem. For each $\alpha>1$, there is a constant $c(\alpha)$ depending on $\alpha$, so that for the $p$-walk on $Q_{n}$ given by (51), we have

$$
\Delta_{T V}(s) \rightarrow 0 \quad \text { as } \quad n \rightarrow \infty
$$

provided $s \geq c(\alpha) n^{\alpha} \log n$

Note that this result is slightly stronger than the corresponding result (48) for (what is essentially) $\alpha=1$. The basic reason for this difference arises from the fact that $\sum_{k=1}^{\infty} k^{-\alpha}$ converges for $\alpha>1$ but diverges for $\alpha=1$.

Proof discussion. The proof of (52) proceeds just like that of (48). The corresponding transition matrix $\bar{Q}$ is decomposed into matrices $\bar{P}_{0}, \bar{P}_{1}, \ldots, \bar{P}_{n / 2}$. As before, $\bar{P}_{0}^{\prime}=I-\bar{P}_{0}$ is the Laplacian on a weighted path $\bar{G}_{n}$, this time on the vertex set $\{0,1, \ldots, n\}$. The degrees and edge weights are now given by

$$
\begin{aligned}
& d_{k}=n(n-1)\left(\begin{array}{l}
n \\
k
\end{array}\right) \\
& w_{k}=(n-k)(n-1) p_{k}\left(\begin{array}{l}
n \\
k
\end{array}\right) .
\end{aligned}
$$

As before, the eigenvalues of $\bar{Q}$ are just the eigenvalues of $\bar{P}_{0}, \bar{P}_{1}, \ldots, \bar{P}_{n / 2}$, with those of $\bar{P}_{k}$ having multiplicity $\left(\begin{array}{c}n \\ k\end{array}\right)-\left(\begin{array}{c}n \\ k-1\end{array}\right)$. We upper bound the eigenvalues of $\bar{P}_{0}$ using a comparison theorem for a "nearby" weighted path $\hat{G}_{n}^{+}$, which has

$$
\hat{d}_{k}= \begin{cases}\frac{n^{2}(n+1)}{n-1} & \text { for } \quad k=0 \\
n(n+1)\left(\begin{array}{l}
n \\
k
\end{array}\right) & \text { for } \quad 1 \leq k \leq n\end{cases}
$$

and

$$
\hat{w}_{k}=(k+2)(n-k)\left(\begin{array}{l}
n \\
k
\end{array}\right) \text { for } \quad 0 \leq k \leq n .
$$

(The difference arises because of the additional vertex 0 in $\bar{G}_{n}$ ). It can be checked that with

$$
\hat{f}(k)=\frac{1}{k+1}-\frac{2}{n+1},
$$

the function $\hat{g}(k)=\hat{f}(k) \sqrt{\hat{d}_{k}}, 0, \leq k \leq n$, is an eigenvector of $\bar{P}_{0}^{\prime}=I-\bar{P}_{0}$ for the eigenvector $\hat{\lambda}_{1}=1 / n$ (which is the smallest positive eigenvalue of $\bar{P}_{0}^{\prime}$ ). The Comparison Lemma then implies

$$
\bar{\lambda}_{1} \geq \frac{1}{2 n}
$$


(where $\bar{\lambda}_{1}$ denotes the smallest positive eigenvalue of $\bar{P}_{0}^{\prime}$ ). With some effort, it can be shown that the maximum row sum $r_{k}$ of $\bar{P}_{k}, k \geq 1$, satisfies

$$
r_{k} \leq 1-\frac{k-1}{n}\left(\frac{k}{n+1}\right)^{n}
$$

(thus upper-bounding any eigenvalue of $\bar{P}_{k}$ ). This is now enough to be able to show that for $y \in V_{n / 2}$,

$$
\begin{aligned}
& \Delta^{\prime}(y, s) \leq \frac{1}{e^{c}-1} \\
& \text { if } s>2 n^{\alpha} \log n+c n^{\alpha}, \quad n>n_{0}(\alpha)
\end{aligned}
$$

(corresponding to (46)). The final calculation is that of estimating $E_{0} T_{n / 2}$ and $E_{n} T_{n / 2}$, the expected times of hitting $n / 2$ starting from either end of $\bar{G}_{n}$ (the larger of which upper bounds $E_{i} T_{n / 2}$ for any $\left.i\right)$. This yields

$$
E_{i} T_{n / 2} \leq c_{0}(\alpha) n^{\alpha}, n \geq n_{0}(\alpha), 0 \leq i \leq n
$$

These results together then combine to give (52).

We remark in closing that it is not hard to derive interpolation results for our walks. The thrust of such results imply that if $0<p_{k} \leq p_{k}^{\prime \prime} \leq p_{k}^{\prime}<1$ for all $k$, then the $p^{\prime \prime}$-walk will mix at least as rapidly as the slower of the $p$-walk and the $p^{\prime}$-walk on $Q_{n}$. This implies for example that if $k / n \leq p_{k}<1, k \geq 0$, then the mixing time of the $p$-walk on $Q_{n}$ is still $O(n \log n)$. 


\section{References}

[A93] D. Aldous, Reversible Markov Chains and Random Walks on Graphs, (University of California at Berkeley, preliminary notes - 1993).

[A94] D. Aldous, (talk at 1994 National IMS meeting).

[C96] F. R. K. Chung, Spectral Graph Theory, CBMS Lecture Notes, Amer. Math. Soc., Providence, 1996.

[CG96] F. R. K. Chung and R. L. Graham, Random walks on generating sets for finite groups, Electronic. J. Combinatorics, 4.2 (1997) \# R7.

[CY95] F.R.K. Chung and S.-T. Yau, Eigenvalues of graphs and Sobolev inequalities, Combinatorics, Probability and Computing, 4 (1995), 11-26.

[DGM90] P. Diaconis, R. L. Graham and J. A. Morrison, Asymptotic analysis of a random walk on a hypercube with many dimensions, Random Structures and Algorithms, 1 (1990), 51-72.

[DS96] P. Diaconis and L. Saloff-Coste, Walks on generating sets of Abelian groups, Prob. Th. and Related Fields, 105 (1996), 393-421.

[GKP94] R. L. Graham, D. E. Knuth and O. Patashnik, Concrete Mathematics 2nd ed., Addison-Wesley, Reading, 1994.

[LT79] G. Letac and L. Takacs, Random walks on the $m$-dimensional cube, J. reine angew. Math, 310 (1979), 187-195. 